\title{
SELFADJOINT FUNCTION SPACES ON RIEMANNIAN SYMMETRIC MANIFOLDS ${ }^{1}$
}

\author{
JUAN A. TIRAO
}

1. Introduction. If $M$ is a compact Hausdorf space then $C(M)$ denotes the Banach algebra (point multiplication, sup norm) of continuous complex valued functions on $M$. A. subspace of $C(M)$ is called selfadjoint if it is closed under complex conjugation of functional values. J. A. Wolf [2] characterized the compact connected Riemannian symmetric manifolds $M$ such that all closed subspaces of $C(M)$, invariant under the largest group of isometries of $M$ are selfadjoint. His result is:

Theorem. All closed G-invariant subspaces of $C(M)$ are selfadjoint if and only if the Weyl group of $M$ contains $-I$ ( $I=$ identity).

The proof given by him is essentially a case by case proof using Cartan's classification of the compact simply connected irreducible Riemannian symmetric spaces.

In this paper, using a different approach, we give a direct proof of that theorem.

I am indebted to J. A. Wolf for suggesting this problem.

2. We assume some familiarity with Riemannian symmetric spaces and their spherical functions, but recall the basic facts in order to establish notation. See Helgason [1] for details.

Let $G$ be a connected Lie group and $K$ a closed subgroup. The pair $(G, K)$ is a symmetric pair if there exists a nontrivial analytic automorphism $\sigma$ of $G$ of order two such that $\left(K_{\sigma}\right)_{0} \subset K \subset K_{\sigma}$, where $K_{\sigma}$ denotes the set of fixed points of $\sigma$ and $\left(K_{\sigma}\right)_{0}$ is the identity component of $K_{\sigma}$. Every connected Riemannian symmetric space $M$ gives rise to a symmetric pair $(G, K)$ where $G$ is the largest connected group of isometries of $M$ and $K$ is the isotropy subgroup of $G$ at some point $x \in M$. Moreover $K$ is compact and $G / K$ is diffeomorphic to $M$. Therefore $M$ is compact if and only if $G$ is compact. From now on we will restrict our attention to a symmetric pair $(G, K)$ where $G$ is compact.

The Lie algebra $\mathcal{G}$ of $G$ is the vector space direct sum $\mathcal{K} \oplus \odot$ where the differential of $\sigma$ is +1 on $\mathscr{K}$ and -1 on $P$, and where $\mathcal{K}$ is the Lie

Received by the editors September 19, 1968.

1 Research supported by a fellowship from the Consejo Nacional de Investigagaciones Científicas y Técnicas de la R. Argentina. 
algebra of $K$. Every subalgebra of $\mathcal{G}$ contained in $P$ is abelian. Let $a$ be a maximal one. It follows that $G=K A K$ where $A=\exp (Q)$. Consider the normalizer $N=\{k \in K: \operatorname{Ad}(k) a \subset Q\}$ of $Q$ in $K$. Conjugation by elements of $N$ induces a finite group of linear transformations of $a$; this is the Weyl group of $(G, K)$.

Let $\pi$ denote the projection map of $G$ onto $G / K$ and $\tau(g)$ the diffeomorphism of $G / K$ given by $\tau(g): x K \rightarrow g x K$. Let $C^{\infty}(G / K)$ denote the algebra of all $C^{\infty}$ complex valued functions on $G / K$. We put $\tilde{f}=f \circ \pi$ if $f \in C(G / K)$, and $\pi(e)=0$. Let $D(G / K)$ denote the algebra of all differential operators on $G / K$ which are invariant under the action of $G$; a differential operator $D$ on $G / K$ belongs to $D(G / K)$ if $D(f \circ \tau(g))=(D f) \circ \tau(g)$ for all $f \in C^{\infty}(G / K), g \in G$.

Let $X_{1}, X_{2}, \cdots, X_{p}$ be a basis of $\beta$ and let $S(\mathcal{P})$ denote the algebra of polynomials in these basis elements. Each endomorphism $T$ of $\beta$ induces a homomorphism $Q \rightarrow T \cdot Q$ of $S(P)$ given by $(T \cdot Q)$ $\left(X_{1}, \cdots, X_{p}\right)=Q\left(T \cdot X_{1}, \cdots, T \cdot X_{p}\right)$. In particular $\hat{Q}$ will denote $(-I) \cdot Q$. Let $I(\mathcal{P})$ denote the set of polynomials in $S(\mathcal{Q})$ which are invariant under $\operatorname{Ad}_{G}(K)$. Note that if $Q \in I(P)$ then $\hat{Q} \in I(P)$.

For $f \in C^{\infty}(G / K)$ and $Q \in I(P)$ the equation

$$
\left(D_{Q} f\right)(\pi(g))=\left(Q\left(\partial_{1}, \cdots, \partial_{p}\right) \tilde{f}\left(g \cdot \exp \left(x_{1} X_{1}+\cdots+x_{p} X_{p}\right)\right)\right)(0)
$$

defines a differential operator on $G / K$ invariant under the action of $G$. The map $Q \rightarrow D_{Q}$ is a one to one linear map of $I(P)$ onto $D(G / K)$. An element $\phi \in C^{\infty}(G / K)$ is called a spherical function on $G / K$ if :

(i) $\phi(\pi(e))=1$,

(ii) $\phi \circ \tau(k)=\phi$ for all $k \in K$,

(iii) $D \phi=\lambda_{\phi}(D) \phi$ for all $D \in D(G / K)$, for certain complex numbers $\lambda_{\phi}(D)$.

3. We start with the following criterion about elements in $I(\mathcal{P})$.

Theorem 3.1. Let $P, Q \in I(\mathcal{P})$. Then $P=Q$ if and only if $\left(D_{P} \phi\right)(0)$ $=\left(D_{Q} \phi\right)(0)$ for all spherical functions $\phi$ on $G / K$.

Proof. Observe that $D_{P} \phi=\left(\left(D_{P} \phi\right)(0)\right) \phi=\left(\left(D_{Q} \phi\right)(0)\right) \phi=D_{Q} \phi$, since $\phi$ is a spherical function. Furthermore $D_{P}(\phi \circ \tau(g))=\left(D_{P} \phi\right) \circ \tau(g)$ $=\left(D_{Q} \phi\right) \circ \tau(g)=D_{Q}(\phi \circ \tau(g))$ for all $g \in G$, since $D_{P}$ and $D_{Q}$ are invariant under $G$. Therefore $D_{P}$ and $D_{Q}$ coincide on each $V_{\phi}$, where $V_{\phi}$ denotes the subspace of $C^{\infty}(G / K)$ spanned by $\{\phi \circ \tau(g): g \in G\}$. If we consider on $C^{\infty}(G / K)$ the $C^{\infty}$-topology (based on uniform convergence of sequences of functions and their successive derivatives), we have the following Fourier-Peter-Weyl developments

$$
C(G / K) \sim \sum V_{\phi}, \quad C^{\infty}(G / K) \sim \sum V_{\phi}
$$


where $\phi$ runs over all the spherical functions on $G / K$. Now the theorem follows from the fact that the differential operators on $G / K$ are continuous linear transformations of $C^{\infty}(G / K)$. Q.E.D.

Let $h$ be a function on $G$. We say that $h$ is bi-invariant under $K$ if $h\left(k g k^{\prime}\right)=h(g)$ for all $k, k^{\prime} \in K$, and we define $\hat{h}$ by $\hat{h}(g)=h\left(g^{-1}\right), g \in G$. Let $f$ be a function on $G / K$ invariant under $K$, i.e. $f \circ \tau(k)=f$ for all $k \in K$. Then the equation $\hat{f}(g K)=f\left(g^{-1} K\right)$ defines a function $\hat{f}$ on $G / K$ such that $\hat{f}^{-}=\tilde{f}^{\hat{n}}$.

Lemma 3.2. Let $f \in C^{\infty}(G / K)$ be invariant under $K$. Then

$$
\left(D_{Q} \hat{f}\right)(0)=\left(D_{\hat{\mathbf{Q}}} f\right)(0)
$$

for all $Q \in I(\odot)$.

PROoF. It is a straight forward computation:

$$
\begin{aligned}
\left(D_{Q} \hat{f}\right)(0) & =\left(Q\left(\partial_{1}, \cdots, \partial_{p}\right) \hat{f} \sim\left(\exp \left(x_{1} X_{1}+\cdots+x_{p} X_{p}\right)\right)\right)(0) \\
& =\left(Q\left(\partial_{1}, \cdots, \partial_{p}\right) \tilde{f}\left(\exp \left(-x_{1} X_{1}-\cdots-x_{p} X_{p}\right)\right)\right)(0) \\
& =\left(\hat{Q}\left(\partial_{1}, \cdots, \partial_{p}\right) \tilde{f}\left(\exp \left(x_{1} X_{1}+\cdots+x_{p} X_{p}\right)\right)\right)(0) \\
& =\left(D_{\hat{Q}} f\right)(0) . \quad \text { Q.E.D. }
\end{aligned}
$$

TheOREM 3.3. Let $(G, K)$ be a symmetric pair, $G$ compact. The following conditions are equivalent:

(i) every closed G-invariant subspace of $C(G / K)$ is selfadjoint;

(ii) the Weyl group of $(G, K)$ contains $-I$;

(iii) every function $f$ on $G$, bi-invariant under $K$, has the property $f(g)=f\left(g^{-1}\right)$ for all $g \in G$;

(iv) the spherical functions on $G / K$ are real valued.

Proof. (i) implies (iv). For every spherical function $\phi, V_{\phi}$ is a closed (in fact $\left.\operatorname{dim} V_{\phi}<\infty\right) G$-invariant subspace of $C(G / K)$ and therefore selfadjoint. Since $\bar{\phi}$ is also a spherical function on $G / K$ and $V_{\phi}$ contains one and only one spherical function, it follows that $\phi=\bar{\phi}$.

(iv) implies (i). If $\phi$ is a real valued spherical function on $G / K$ then all the translates $\phi \circ \tau(g),(g \in G)$, of $\phi$ are real valued, hence $V_{\phi}$ is selfadjoint. Using the Fourier-Peter-Weyl development of $C(G / K)$ the assertion follows.

(ii) implies (iii). Let $f$ be a function on $G$ bi-invariant under $K$. By hypothesis there exists an element $k \in K$ such that $\operatorname{Ad}(k) H=-H$ for all $H \in Q$. Therefore if $a=\exp H, H \in Q$, we have

$$
f(a)=f(\exp H)=f\left(k(\exp H) k^{-1}\right)=f(\exp (-H))=f\left(a^{-1}\right)
$$

for all $a \in A$. Using the fact that $G=K A K$ we get 


$$
f(g)=f\left(k a k_{1}\right)=f(a)=f\left(a^{-1}\right)=f\left(k_{1}^{-1} a^{-1} k^{-1}\right)=f\left(g^{-1}\right)
$$

for all $g \in G$.

(iii) implies (iv). If $\phi$ is a spherical function on $G / K$ it follows that $\Phi$ is a positive definite function on $G$, i.e.,

$$
\sum_{i, j=1}^{n} \delta\left(g_{i}^{-1} g_{j}\right) \alpha_{i} \bar{\alpha}_{j} \geqq 0
$$

for all finite sets $g_{1}, \cdots, g_{n}$ of elements in $G$ and any complex numbers $\alpha_{1}, \cdots, \alpha_{n}$. In particular we have therefore that $\tilde{\phi}^{\wedge}=\tilde{\phi}^{-}$. Since $\tilde{\phi}$ is bi-invariant under $K, \tilde{\phi}=\tilde{\phi}^{\wedge}=\tilde{\phi}^{-}=\bar{\phi}^{-}$; hence $\phi=\bar{\phi}$.

(iv) implies (ii). If $\phi$ is a real valued spherical function on $G / K$, then $\tilde{\phi}=\tilde{\phi}^{\wedge}$ and so $\hat{\phi}^{-}=\tilde{\phi}^{\wedge}=\tilde{\phi}$; hence $\phi=\hat{\phi}$. From Lemma $3.2 \mathrm{it}$ follows that

$$
\left(D_{Q} \phi\right)(0)=\left(D_{Q} \hat{\phi}\right)(0)=\left(D_{\hat{Q}} \phi\right)(0)
$$

for all spherical functions on $G / K$. Therefore Theorem 3.1 implies that $Q=\hat{Q}$ for all $Q \in I(P)$. Given $H \in \odot$ consider the compact orbits $\operatorname{Ad}_{G}(K) H$ and $\operatorname{Ad}_{G}(K)(-H)$. If these two orbits were disjoint it would be possible to find a continuous function on $P$ identically 1 on $\operatorname{Ad}_{G}(K)(-H)$ and identically 0 on $\operatorname{Ad}_{G}(K) H$. Owing to the Weierstrass approximation theorem there would exist a polynomial $Q \in S(\mathcal{P})$ such that

$$
\begin{array}{rlrl}
|Q(X)| & \leqq 1 / 3 & & \text { for all } X \in \operatorname{Ad}_{G}(K) H \\
|Q(X)-1| & \leqq 1 / 3 & \text { for all } X \in \operatorname{Ad}_{G}(K)(-H) .
\end{array}
$$

Then the polynomial

$$
Q_{0}=\int_{K} \operatorname{Ad}_{G}(k) \cdot Q d k
$$

( $d k$ normalized Haar measure on $K$ ), would belong to $I(\beta)$ and would take different values at $H$ and $-H$. This would contradict the fact that every $Q \in I(P)$ has the property $Q(X)=\hat{Q}(X)=Q(-X)$ for all $X \in \mathcal{P}$. Hence for any $H \in \mathcal{P}, \operatorname{Ad}_{G}(K) H$ intersects $\operatorname{Ad}_{G}(K)(-H)$, in particular, for any $H$ there exists $k \in K$ such that $\operatorname{Ad}_{G}(k) H=-H$. It is always true that given a subset $B$ of $Q$ such that $\operatorname{Ad}_{G}(k) B \subset Q$ for some $k \in K$, then there exists an element $s$ in the Weyl group $W$ of $(G, K)$ such that $s \cdot H=\operatorname{Ad}_{G}(k) H$ for all $H \in ß$. Therefore there exists an element $s \in W$ such that $s \cdot H=-H$. In other words, if $A_{s}$ denotes the linear subspace of $a$ of all $H \in Q$ such that $s \cdot H=-H, s \in W$, we have proved that $a=\bigcup_{s \in W} A_{s}$. Since $W$ is finite, it follows that for some $s \in W, A_{8}=a$. This completes the proof. Q.E.D. 
As a corollary of Theorem 3.3 we point out the corresponding result for compact connected Lie groups.

Given a compact connected Lie group $G$ let $T(G)$ denote the group of transformations of $G$ generated by the right and left translations.

THEOREM 3.4. Let $G$ be a compact connected Lie group. The following conditions are equivalent:

(i) every closed $T(G)$-invariant subspace of $C(G)$ is selfadjoint;

(ii) the Weyl group of $G$ contains $-I$;

(iii) every element of $G$ is conjugate to its inverse;

(iv) every function $f$ on $G$ which is constant on conjugacy classes has the property $f(g)=f\left(g^{-1}\right)$ for all $g \in G$;

(v) the characters of all irreducible representations of $G$ are real.

Proof. If $T$ is a maximal toral subgroup of $G$, then the Weyl group of $G$ is the group of linear transformations of the Lie algebra of $T$ induced from conjugation by elements in the normalizer of $T$ in $G$. Let $K$ denote the diagonal of $G \times G$ then $(G \times G, K)$ is a symmetric pair (the symmetry is $\left.\sigma:\left(g_{1}, g_{2}\right) \rightarrow\left(g_{2}, g_{1}\right)\right)$ and $(G \times G) / K$ is diffeomorphic to $G$ under the mapping

$$
\left(g_{1}, g_{2}\right) K \rightarrow g_{1} g_{2}^{-1} \quad g_{1}, g_{2} \in G .
$$

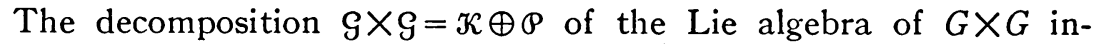
duced by the differential of $\sigma$ is $\mathscr{K}=\{(X, X): X \in \mathcal{G}\}$ and $\beta=$ $\{(X,-X): X \in \mathcal{G}\}$. The mapping $X \rightarrow(X,-X)$ maps $\mathcal{G}$ onto $\odot$, mapping the Lie algebra of $T$ onto a maximal abelian subalgebra of

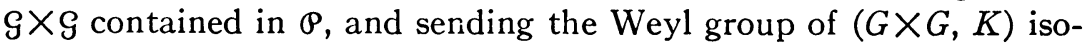
morphically on to the Weyl group of $G$.

The only thing we have to add is that from the theory of representations of compact groups it is known that the spherical functions on $G$ are precisely

$$
\phi=\left(1 / d_{x}\right) x
$$

where $\chi$ is the character of an irreducible representation of $G$ and $d_{x}$ denotes its dimension. Q.E.D.

Corollary 3.5. If the Weyl group of $G$ contains $-I$, then $G$ is semisimple and none if its simple components is locally isomorphic to $S U(n)(n>2)$, to $S O(4 n+2)$, nor to $E_{6}$.

Proof (Cf. [2, §4.8]). The Lie algebra of $G$ is compact. Thus $\mathcal{G}=\mathrm{Z} \oplus[\mathcal{G}, \mathcal{G}$ ] where $\mathrm{Z}$ is the center of $\mathcal{G}$ and $[\mathcal{G}, \mathcal{G}]$ is semisimple. From Theorem 3.4 (iii) every central element of $G$ has square 1, 
therefore $G$ can not be locally isomorphic to a Lie group with a vector subgroup as direct factor, hence $Z=\{0\}$ and $G$ is semisimple. The universal covering group $\tilde{G}$ of $G$ is compact (Weyl theorem) and the Weyl group of $\tilde{G}$ also contains $-I$, therefore every central element of $G$ has square 1 . If a simple component of $G$ is locally isomorphic to $\mathrm{SU}(n)(n>2)$, to $\mathrm{SO}(4 n+2)$ or to $E_{6}$, then $G$ has a direct factor $\mathrm{SU}(n)(n>2)$, or $\mathrm{SO}(4 n+2)$, or $E_{6}$, respectively, which is impossible because the center of that direct factor is cyclic of order $n>2$, or cyclic of order 4 , or cyclic of order 3 , respectively. Q.E.D.

REMARK 3.6. From Cartan classification of compact simple simply connected Lie groups it follows that if $G$ is a compact connected semisimple Lie group such that none of its simple components is locally isomorphic to $\mathrm{SU}(n)(n>2)$, to $\mathrm{SO}(4 n+2)$, nor to $E_{6}$, then the Weyl group of $G$ contains $-I$.

\section{REFERENCES}

1. S. Helgason, Differential geometry and symmetric spaces, Academic Press, New York, 1962.

2. J. A. Wolf, Selfadjoint function spaces on Riemannian symmetric manifolds. Trans. Amer. Math. Soc. 113 (1964), 299-315.

Universidad Nacional de Córdoba, Argentina 